\title{
Efeitos Observados com Diferentes Doses de Morfina Subaracnóidea em Ratos
}

\section{Effects of Different Spinal Morphine Doses in Rats}

Neuzimar de Souza Freire Silva ${ }^{1}$; Rioko Kimiko Sakata, TSA ${ }^{2}$; Adriana Machado Issy ${ }^{2}$

\author{
RESUMO \\ Silva NSF, Sakata RK, Issy AM - Efeitos Observados com Dife- \\ rentes Doses de Morfina Subaracnóidea em Ratos
}

\begin{abstract}
JUSTIFICATIVA E OBJETIVOS: A morfina por via espinhal promove bom efeito analgésico, mas não é isenta de efeito colateral. O objetivo deste estudo foi investigar os efeitos observados com diferentes doses de morfina por via subaracnóidea.

MÉTODO: Foram estudados cinco grupos de sete ratos, 24 horas após colocação de cateter subaracnóideo via cisterna magna sob anestesia com cetamina e xilazina por via muscular. O G1 recebeu $10 \mu$ lde solução fisiológica; os grupos G2, G3, G4, e G5 receberam respectivamente 0,1; 0,3; 0,5 e $1 \mu \mathrm{g}$ de morfina em $10 \mu$ de solução fisiológica. Os animais foram submetidos ao teste de imersão da cauda em água quente no $M_{0}$ (antes da injeção), e $M_{15}, M_{30}, M_{60}, M_{120}$ e $M_{180}$ minutos após injeção.
\end{abstract}

RESULTADOS: Foi observada analgesia nos grupos que receberam morfina, em diversos momentos, quando comparados com o grupo controle e com o tempo antes da injeção de morfina. No $G 1$ houve fraqueza das patas em 4 animais. Agitação ocorreu em $M_{15}$ no $G 2$ e em $M_{15}$ e $M_{30}$ no G3. Tremor mandibular foi observado em $M_{5}, M_{15}, M_{30}$ e $M_{60}$ no $G 2$; no $G 3$ foi observado em $M_{5}$ e $M_{15}$; no $G 4$, em $M_{5}$ e no G5, em $M_{5}$. Prurido foi observado em $M_{5}, M_{15}, M_{30}$ e $M_{60}$ no $G 2$; em $M_{5}, M_{15}$, $M_{30}, M_{60}$ e $M_{120}$ no G3; em $M_{5}, M_{15}, M_{30}, M_{60}$, e $M_{120}$ no G4; em $M_{5}, M_{15}, M_{30}, M_{60}$, e $M_{120}$ no G5. Ausência de diurese ocorreu em $M_{60}$ no G2; no $M_{15}, M_{30}, M_{60}$ e $M_{120}$ no G3; no $M_{60}$ e $M_{120}$ no G4; e no $M_{30}$ e $M_{60}$ no G5. Sedação ocorreu em $M_{15}, M_{30}$ e $M_{60}$ nos grupos 2, 3 e 4; e em $M_{15}, M_{30}, M_{60}$ e $M_{120}$ no $G 5$. Alteração respiratória foi observada em $M_{15}, M_{30}, M_{60}$ no G2; em $M_{15}, M_{30}$ e $M_{60}$ no G3; em $M_{15}$ e $M_{30}$ nos grupos 4 e 5 .

CONCLUSÕES: Neste estudo, todas as doses de morfina subaracnóideas administradas provocaram efeitos colaterais; e doses menores que $1 \mathrm{\mu g}$ promoveram analgesia de curta duração.

Unitermos: ANALGÉSICOS, Opióides: morfina; ANIMAL: rato; TÉCNICAS ANESTÉSICAS, Regional: subaracnóidea

\footnotetext{
* Recebido da (Received from) Disciplina de Anestesiologia, Dor e Terapia Intensiva da Universidade Federal de São Paulo (UNIFESP), São Paulo, SP

1. Pós-Graduando da Disciplina de Anestesiologia, Dor e Terapia Intensiva da UNIFESP

2. Professora Adjunta de Anestesiologia da UNIFESP
}

Apresentado (Submitted) em 04 de fevereiro de 2003

Aceito (Accepted) para publicação em 06 de maio de 2003

Endereço para correspondência (Correspondence to)

Dra. Rioko Kimiko Sakata

Rua Três de Maio 61/51 Vila Clementino

04044-020 São Paulo, SP

E-mail: riokoks.dcir@epm.br

(C) Sociedade Brasileira de Anestesiologia, 2004

\author{
SUMMARY \\ Silva NSF, Sakata RK, Issy AM- Effects of Different Spinal Morp- \\ hine Doses in Rats
}

BACKGROUND AND OBJECTIVES: Spinal morphine promotes adequate pain relief, but is not free from side effects. This study aimed at investigating the effects of different spinal morphine doses.

METHODS: Five groups of seven rats were studied, $24 \mathrm{~h}$ after spinal catheter insertion via cisterna magna under anesthesia with muscular ketamine and xylazine. G1 received $10 \mu$ saline solution; groups $\mathrm{G} 2, \mathrm{G} 3, \mathrm{G} 4$ and $\mathrm{G} 5$ received respectively 0.1 ; $0.3 ; 0.5$ and $1 \mu \mathrm{g}$ morphine in $10 \mu \mathrm{l}$ saline solution. Animals were submitted to tail immersion test in hot water at $M_{0}$ (prior to injection), and $M_{15}, M_{30}, M_{60}, M_{120}$ and $M_{180}$ minutes after the injection.

RESULTS: Analgesic effects were observed in groups receiving morphine, in different times, as compared to control and to time before morphine injection. In $\mathrm{G} 1$ there was paws weakness in 4 animals. Agitation was observed at $M_{15}$ in $G 2$ and at $M_{15}$ and $M_{30}$ in G3. Mandible tremors were observed at moments $M_{5}$, $M_{15}, M_{30}$ and $M_{60}$ in $G 2$; at $M_{5}$ and $M_{15}$ in $G 3$, in $M_{5}$ in $G 4$, and in $M_{5}$ in G5. Pruritus was observed at $M_{5}, M_{15}, M_{30}$ and $M_{60}$ in $G 2$; at $M_{5}, M_{15}, M_{30}, M_{60}$ and $M_{120}$ in $G 3$; at $M_{5}, M_{15}, M_{30}, M_{60}$, and $M_{120}$ in G4; and at $M_{5}, M_{15}, M_{30}, M_{60}$ and $M_{120}$ in G5. Absence of diuresis was observed at $M_{60}$ in G2; at $M_{15}, M_{30}, M_{60}$ and $M_{120}$ in G3; at $M_{30}$ and $M_{120}$ in $G 4$, and at $M_{30}$, and $M_{60}$ in $G 5$. Sedation was observed at $M_{15}, M_{30}$ and $M_{60}$ in groups 2, 3 and 4; and at $M_{15}, M_{30}$, $M_{60}$ and $M_{120}$ in G5. Respiratory changes were observed at $M_{15}$, $M_{30}$ and $M_{60}$ in G2; at $M_{15}, M_{30}$ and $M_{60}$ in $G 3$; at $M_{15}$ and $M_{30}$ in groups 4 and 5 .

CONCLUSIONS: In this study all morphine doses below $1 \mu \mathrm{g}$ have promoted short duration analgesia; all spinal morphine doses have produced side effects.

Key Words: ANALGESICS, Opioids: morphine; ANIMAL: rat, ANESTHETIC TECHNIQUES, Regional: spinal block

\section{INTRODUÇÃO}

Com o conhecimento das propriedades farmacológicas dos analgésicos administrados por diferentes vias, tornou-se possível melhorar o tratamento da dor, reduzindo as complicações decorrentes de diversas síndromes dolorosas.

As pesquisas realizadas em laboratório experimental nos forneceram as bases para a utilização clínica de opióides no homem, com segurança e eficácia no controle da dor agudae crônica. Após a descoberta de receptores opióides em 1974 ${ }^{1}$, foi obtida analgesia de longa duração com morfina por via subaracnóidea em ratos ${ }^{2}$ e no homem ${ }^{3}$. Desde então, esses fármacos vêm sendo empregados com freqüência por via espinhal para alívio da dor aguda e crônica.

A morfina é um opióide hidrofílico que promove analgesia intensa e de longa duração, sem provocar bloqueios simpático 
ou motor. Entretanto, apresenta dispersão cranial, podendo provocar efeitos colaterais como prurido, náusea, vômito e insuficiência respiratória.

Para tentar verificar se é possível efeito analgésico com morfina por via subaracnóidea isenta de efeitos colaterais foram administradas diferentes doses observando os animais, em diferentes momentos.

\section{MÉTODO}

Este estudo experimental foi realizado após ter sido aprovado pelos Comitês de Ética em Pesquisa da Universidade Federal de São Paulo e da Universidade Federal do Amazonas. Foram investigados 35 ratos da linhagem Wistar, machos, sadios, com idade aproximada de 90 dias e peso corporal entre 250 e 300 gramas. Foram excluídos do protocolo, ratos com paralisia ou flacidez das patas. Os animais foram mantidos em gaiolas individuais, recebendo 12 horas de luz por dia, água e ração à vontade durante todo o experimento. Os animais foram distribuídos em 5 grupos de 7 e todos receberam $10 \mu$ l de solução por via subaracnóidea: os do G1 (controle), receberam $10 \mu$ lde solução fisiológica a $0,9 \%$; os do G2, 0,1 $\mu$ g de morfina; os do G3, 0,3 $\mu$ g de morfina; os do G4, $0,5 \mu \mathrm{g}$ de morfina; e os do $\mathrm{G} 5,1 \mu \mathrm{g}$ de morfina.

Para preparo das soluções, foi diluído $1 \mathrm{mg}$ do sal de sulfato de morfina em $1 \mathrm{ml}$ de solução fisiológica a 0,9\% (1 mg/ml). Para a solução de $0,1 \mu \mathrm{g} / 10 \mu \mathrm{l}$ foram adicionados $4950 \mu \mathrm{l}$ de solução fisiológica a $0,9 \%$ em $50 \mu \mathrm{l}$ da solução inicial. Para solução de $0,3 \mu \mathrm{g} / 10 \mu \mathrm{l}$, foram adicionados $3000 \mu \mathrm{l}$ de solução fisiológica a 0,9\% em $100 \mu \mathrm{l}$. Para solução de $0,5 \mu \mathrm{g} / 10 \mu \mathrm{l}$ foram adicionados $2000 \mu \mathrm{l}$ de solução fisiológica a $0,9 \%$ em $100 \mu \mathrm{l}$. Para solução de $1 \mu \mathrm{g} / 10 \mu \mathrm{l}$, foram adicionados $4500 \mu \mathrm{l}$ de solução fisiológica a $0,9 \%$ em $500 \mu$ l.

A colocação de cateter subaracnóideo foi feita pela técnica de Yaksh, Rudy (1976), modificada, sob anestesia geral com cetamina $\left(60 \mu \mathrm{g} \cdot \mathrm{g}^{-1}\right)$ exilazina $\left(16 \mu \mathrm{g} \cdot \mathrm{g}^{-1}\right)$ por via muscular.

Aextremidade livre do cateter foi exteriorizada na pele, entre o occipital e a coluna cervical alta e fixada com acrílico polimerizado e fio de nylon.

Os animais foram mantidos em gaiolas individuais para recuperação anestésica durante 24 horas e os com deficiência neurológica foram excluídos do estudo. Os animais foram submetidos ao teste de analgesia através da imersão da cauda em água quente a temperatura de $50 \pm 0,2^{\circ} \mathrm{C}$. Para realização deste teste, as caudas dos animais receberam uma marca com tinta esmalte vermelha a $5 \mathrm{~cm}$ da extremidade, para imersão. Os animais foram colocados em um tubo cilíndrico de plástico semi-transparente, para impedir a livre movimentação. Foi avaliado o tempo para retirada da cauda não mantendo a cauda mais que 15 segundos.

Após o teste de analgesia no $\mathrm{T}_{0}$ (antes da morfina), foram administradas aleatoriamente diferentes concentrações de morfina, 24 horas após a colocação do cateter no espaço subaracnóideo. Todos os animais que participaram do estudo estavam ativos, movimentando-se, recebendo ração e água livres; totalmente recuperados da anestesia.
A injeção pelo cateter foi efetuada com seringa de Hamilton de $10 \mu \mathrm{l}$, graduada de 1 em $1 \mu \mathrm{l}$, em uma velocidade de $5 \mu \mathrm{l}$ por segundo.

O teste de analgesia foi realizado nos momentos: $M_{0}=$ antes da administração da morfina; $\mathrm{M}_{15}=15$ minutos após a administração da morfina; $M_{30}=30$ minutos após a administração da morfina; $M_{60}=60$ minutos após a administração da morfina; $M_{120}=120$ minutos após a administração da morfina e $\mathrm{M}_{180}=180$ minutos após a administração da morfina.

Foram observados e anotados os possíveis efeitos colaterais e complicações nos momentos $M_{0}, M_{5}, M_{15}, M_{30}, M_{60}, M_{120}$ e $\mathrm{M}_{180}$.

Para análise estatística foram empregados os testes não-paramétricos. AAnálise de Variância foi utilizada para o estudo da analgesia pelo teste de imersão da cauda, para comparar o grupo controle em cada momento avaliado e os momentos em cada grupo. Para todos os teste, foi fixado em 0,05 ou $5 \%$ $(p \leq 0,05)$ o nível de rejeição da hipótese de nulidade.

\section{RESULTADOS}

\section{Efeito Analgésico}

$O$ efeito analgésico não foi avaliado no $M_{5}$ em nenhum dos grupos. Houve diferença estatisticamente significante na analgesia avaliada pelo tempo de retirada da cauda entre os animais que receberam morfina e os do grupo controle (G1). No $G 2$, o tempo para retirada da cauda foi maior que no $G 1$ em $M_{15}$; no $G 3$, em $M_{30}, M_{60}$ e $M_{120}$; no G4, em $M_{15}, M_{30}, M_{60}$, e $M_{120}$; e no $G 5$, em $M_{15}, M_{30}, M_{60}, M_{120}$, e $M_{180}$ (Tabela I).

Tabela I - Valores das Médias dos Tempos para Retirada da Cauda nos Grupos (G1: controle, G2: 0,1 1 g; G3: 0,3

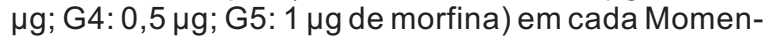
to $\left(M_{0}, M_{15}, M_{30}, M_{60}, M_{120}\right.$ e $\left.M_{180}\right)$

\begin{tabular}{lccccc}
\hline Momentos & \multicolumn{5}{c}{ Grupos } \\
\hline & $\mathrm{G} 1$ & $\mathrm{G} 2$ & $\mathrm{G} 3$ & $\mathrm{G} 4$ & $\mathrm{G} 5$ \\
\hline $\mathrm{M}_{0}$ & 8,66 & 8,14 & 9,29 & 8,43 & 10,57 \\
$\mathrm{M}_{15}$ & 9,86 & $12,86^{*}$ & 11,71 & $13,14^{*}$ & $14,00^{*}$ \\
$\mathrm{M}_{30}$ & 9,71 & 12,00 & $12,71^{*}$ & $14,86^{*}$ & $15,00^{*}$ \\
$\mathrm{M}_{60}$ & 9,14 & 12,57 & $12,86^{*}$ & $13,29^{*}$ & $13,86^{*}$ \\
$\mathrm{M}_{120}$ & 8,86 & 8,86 & $11,57^{*}$ & $12,43^{*}$ & $12,43^{*}$ \\
$M_{180}$ & 8,57 & 8,14 & 9,00 & 9,57 & $11,00^{*}$ \\
\hline
\end{tabular}

$\mathrm{M}_{0}: \mathrm{G} 1=\mathrm{G} 2=\mathrm{G} 3=\mathrm{G} 4=\mathrm{G} 5 ; \mathrm{M}_{15}: \mathrm{G} 2, \mathrm{G} 4$ e G5 $>\mathrm{G} 1=\mathrm{G} 3 ; \mathrm{M}_{30}: \mathrm{G} 3, \mathrm{G} 4 \mathrm{e}$ $\mathrm{G} 5>\mathrm{G} 1=\mathrm{G} 2 ; \mathrm{M}_{60}$ : G3, G4 e G5 > G1 = G2; $\mathrm{M}_{120}$ : G3, G4 e G5 > G1 = $\mathrm{G} 2 ; \mathrm{M}_{180}: \mathrm{G} 5>\mathrm{G} 1=\mathrm{G} 2=\mathrm{G} 3=\mathrm{G} 4$.

* = significância estatística; Análise de Variância $(p \leq 0,05)$

No $\mathrm{G} 2$, foi observado efeito analgésico em $85,72 \%$ dos ratos em $M_{15}$; e 71,43\% em $M_{30}$ e $M_{60}$. No G3, em 71,43\% em $M_{15}$; $100 \%$ em $M_{30}$ e $M_{60}$ e $14,28 \%$ em $M_{120}$. No G4, em $100 \%$ dos animais em $\mathrm{M}_{15}, \mathrm{M}_{30}$ e $\mathrm{M}_{60} ; 71,43 \%$ em $\mathrm{M}_{120}$. No $\mathrm{G} 5$, em $100 \%$ em $M_{15}, 85,72 \%$ em $M_{30}$ e $100 \%$ em $M_{60}$.

Não houve diferença estatisticamente significante na média dos tempos para retirada da cauda entre os momentos $\left(\mathrm{M}_{0}\right.$, 
$M_{15}, M_{30}, M_{60}, M_{120}$ e $M_{180}$ ) no grupo controle. Nos grupos 2, 3 e 5, em $M_{15}, M_{30}$ e $M_{60}$ foram maiores que em $M_{120}$ e $M_{180}$ que foram semelhantes a $M_{0}$. No $G 4$, em $M_{15}, M_{30}, M_{60}$ e $M_{120}$ foram maiores que em $\mathrm{M}_{180}$, que foi semelhante com $\mathrm{M}_{0}$; Análise de Variância, $p \geq 0,05$ (Tabela II).

Tabela II - Valores das Médias dos Tempos para Retirada da Cauda nos Diferentes Períodos em cada Grupo (G1: controle, G2: 0,1 $\mathrm{gg}$; G3: 0,3 $\mu \mathrm{g} ; \mathrm{G} 4: 0,5 \mu \mathrm{g} ; \mathrm{G} 5: 1 \mu \mathrm{g}$ de morfina)

\begin{tabular}{lcccccc}
\hline Grupos & \multicolumn{5}{c}{ Momentos após Administração das Soluções } \\
& $M_{0}$ & $M_{15}$ & $M_{30}$ & $M_{60}$ & $M_{120}$ & $M_{180}$ \\
\hline G1 $(n=7)$ & 8,66 & 9,86 & 9,71 & 9,14 & 8,86 & 8,57 \\
G2 $(n=7)$ & 8,14 & $12,86^{*}$ & $12,00^{*}$ & $12,57^{*}$ & 8,86 & 8,14 \\
G3 (n=7) & 9,29 & $11,71^{*}$ & $12,71^{*}$ & $12,86^{*}$ & 11,57 & 9,00 \\
G4 (n=7) & 8,43 & $13,14^{*}$ & $14,86^{*}$ & $13,29^{*}$ & $12,43^{*}$ & 9,57 \\
G5 (n=7) & 10,57 & $14,00^{*}$ & $15,00^{*}$ & $13,86^{*}$ & 12,43 & 11,00 \\
\hline
\end{tabular}

$G 2: M_{15}, M_{30}$ e $M_{60}>M_{0}=M_{120}=M_{180} ; G 3: M_{15}, M_{30}$ e $M_{60}>M_{0}=M_{120}=$ $M_{180} ; G 4: M_{15}, M_{30}, M_{60}$ e $M_{120}>M_{0}=M_{180} ; G 5: M_{15}, M_{30}$ e $M_{60}>M_{0}=M_{120}$ $=\mathrm{M}_{180}$;

*:significância estatística; Análise de Variância $(p \leq 0,05)$

\section{Efeitos Colaterais Observados após Injeção das Soluções}

No G1 não houve manifestação de nenhum efeito exceto no $\mathrm{M}_{5}$, em que ocorreu fraqueza das patas em quatro $(57,15 \%)$ animais.

Agitação foi observada no $\mathrm{G} 2$ em $14,28 \%$ dos animais em $M_{15}$; no $G 3$, em $14,28 \%$ em $M_{15}$ e $M_{30}$.

Foi observado tremor mandibular no $\mathrm{G} 2$ em $85,72 \%$ dos animais em $M_{5} ; 71,43 \%$ em $M_{15}$ e 14,28\% em $M_{30}$ e $M_{60}$. No G3, foi observado esse efeito colateral em $100 \%$ dos animais em $M_{5}$ e $14,28 \%$ em $M_{15}$. No G4, foi observado em $85,72 \%$ dos animais em $\mathrm{M}_{5}$. No G5, foi observado em 85,72\% dos animais em $\mathrm{M}_{5}$.

Prurido ocorreu no $\mathrm{G} 2$ em $57,15 \%$ dos animais em $\mathrm{M}_{5}$; $71,43 \%$ em $M_{15} ; 57,15 \%$ em $M_{30}$; e $42,86 \%$ em $M_{60}$. No G3, esse efeito foi observado em $42,86 \%$ em $\mathrm{M}_{5}, 85,72 \%$ em $\mathrm{M}_{15}$; $100 \%$ em $M_{30}$ e $M_{60}$ e $57,15 \%$ em $M_{120}$. No G4, foi observado em $85,72 \%$ em $M_{5}, 100 \%$ dos animais em $M_{15}, M_{30}$ e $M_{60}$ e $71,43 \%$ em $M_{120}$. No $G 5$, foi observado $71,43 \%$ de prurido em $M_{5}, 100 \%$ em $M_{15}, M_{30}, M_{60}$ e $28,58 \%$ em $M_{120}$.

Foi observada ausência de diurese no G2 em $85,72 \%$ dos animais em $M_{60}$. No G3, foi observado esse efeito em 14,28\% em $\mathrm{M}_{15}$ e $\mathrm{M}_{30} ; 71,43 \%$ em $\mathrm{M}_{60}$ e $28,58 \%$ em $\mathrm{M}_{120}$. No G4, foi observada ausência de diurese em 85,72\% em $\mathrm{M}_{60}$ e 28,58\% em $\mathrm{M}_{120}$. No $\mathrm{G} 5$, foi observada ausência de diurese em $14,28 \%$ dos animais em $M_{30}$ e $100 \%$ em $M_{60}$.

Foi observada sedação no $\mathrm{G} 2$ em $100 \%$ dos animais em $\mathrm{M}_{15}$, $85,72 \%$ em $M_{30}$ e $57,15 \%$ em $M_{60}$. No G3, foi observado o mesmo efeito em $57,15 \%$ em $M_{15}, 85,72 \%$ em $M_{30}$ e $57,15 \%$ em $M_{60}$. No G4, observou-se sedação em $100 \%$ dos animais em $M_{15}$ e $M_{30}$ e 71,43\% em $M_{60}$. No G5, observou-se sedação em $100 \%$ dos animais em $M_{15}$ e $M_{30}$, em $57,15 \%$ em $M_{60}$ e $14,28 \%$ em $\mathrm{M}_{120}$.
Ocorreu alteração respiratória em G2 em 71,43\% dos animais em $M_{15}$ e $M_{30}$, e 42,86\% em $M_{60}$. No G3, houve alteração respiratória em 42,86\% dos animais em $\mathrm{M}_{15}, 14,28 \%$ em $\mathrm{M}_{30} \mathrm{e}$ $M_{60}$. No G4, esse efeito ocorreu em $100 \%$ dos animais em $M_{15}$ e $M_{30}$. No G5, ocorreu alteração respiratória em 42,86\% em $M_{15}$ e $57,15 \%$ em $M_{30}$.

\section{DISCUSSÃO}

Para este estudo foi escolhido o rato Wistar, não só pela facilidade de aquisição, devido à rapidez de reprodução e pelo pequeno porte, tornando-se fácil seu manuseio e a manutenção da alimentação e da higiene nas gaiolas, grande resistência às infecções e baixo custo. Muitos autores também optaram por ratos para injeção de morfina por via subaracnóidea, pelos motivos descritos ${ }^{4,5}$.

O opióide escolhido foi a morfina, por ser um analgésico potente, usado por via espinhal, principalmente para o tratamento da dor aguda e também para síndromes dolorosas crônicas. Por ser hidrofílico, sua duração de ação é prolongada, porém essa característica também é responsável por permanência do fármaco no líquor durante tempo maior que com opióide lipofílico. Com isso, ocorrem maior difusão cranial e ligação do opióide aos receptores encefálicos, provocando efeitos colaterais como prurido, náusea, vômito e depressão respiratória. A presença desses efeitos colaterais limita o uso de opióides por via espinhal, portanto, se houver a possibilidade de administração de uma dose que produza efeito analgésico sem efeitos colaterais, seu uso poderia ser ampliado.

Nas primeiras investigações havia questionamento se alguma quantidade do opióide alcançava estruturas encefálicas imediatamente após a injeção. Inicialmente imaginou-se que a droga se moveria no sentido cranial, passivamente seguindo o movimento do líquor dentro do espaço subaracnóideo. Após administração de diferentes volumes de bromofenol azul no espaço subaracnóideo de ratos, seguida de estudo da medula espinhal ${ }^{4}$, os autores concluíram que volume de $10 \mu$ é o mais adequado. Outros autores utilizaram volumes de 5 a $20 \mu l^{6}$, até $100 \mu l^{7}$ por via peridural e subaracnóidea. Porém, um autor comparou diferentes volumes por via subaracnóidea, concluindo que com $10 \mu$ locorreu melhor difusão e afirmou ainda que grandes volumes provocam perda por refluxo e pelas laterais do cateter ${ }^{8}$. Os estudos mostram que não há necessidade de grandes volumes para a morfina exercer efeito analgésico por via espinhal. Diante dos estudos mencionados, foram escolhidos $10 \mu$ l para esta pesquisa por ser o volume que mostrou proporcionar melhor uniformidade quanto à difusão da droga dentro do espaço subaracnóideo e pela segurança.

Foram escolhidas doses de 0,1 a $1 \mu \mathrm{g}$, porque estudo mostrou que $1 \mu \mathrm{g}$ de morfina, por via subaracnóidea, produz efeito analgésico ${ }^{8}$, porém essa dose provoca efeitos colaterais; portanto, tivemos necessidade de empregar doses menores para verificar se há efeito analgésico sem efeito colateral. Alguns autores avaliaram a concentração extracelular de morfina na medula espinhal, substância cinzenta periaque- 
dutal, núcleo da rafe e hipotálamo lateral, após uma dose de morfina de 5 e $10 \mathrm{mg}$ intraperitonial. Na presente pesquisa as doses de morfina administradas promoveram analgesia em muitos tempos dos grupos estudados, porém na maioria dos tempos, a concentração de morfina no líquor foi menor que a concentração mínima de 200 a 400pg/100 $\mu$ l, relatada na literatura ${ }^{9}$.

Para este trabalho, optou-se pelo cateter de borracha de silicone, por ser constituído de material flexível, inerte, atóxicoe não causar reação inflamatória nos tecidos. Tais características foram comprovadas por estudos histopatológicos de medula espinhal de ratos, que permaneceram com cateteres de borracha de silicone implantados durante quatro semanas ${ }^{10}$. Foi escolhido cateter de pequeno diâmetro para este estudo para evitar compressão da medula espinhal.

Para colocação do cateter, foi utilizada a técnica cirúrgica descrita por Yaksh, Rudy ${ }^{4}$ modificada, por permitir acesso relativamente fácil ao espaço subaracnóideo através da cisterna magna. Também possibilita a manutenção do cateter durante períodos prolongados, porque permite sua fixação eficaz, além de isolá-lo do contato com a boca e as patas do animal, impedindo-o de retirá-lo. O método tem sido usado com sucesso para estudar a eficácia de agentes injetados por via subaracnóidea. Entretanto, se reproduzida fielmente, demanda um tempo muito grande para a colocação do cateter e apresenta um elevado índice de falha para a coleta de líquor.

Neste trabalho, inicialmente, a punção da membrana foi feita como os autores recomendam, com a punção no meio da membrana atlanto-occipital, mas não houve sucesso na coleta seriada de líquor. Com modificação e introdução do cateter na parte mais lateral (junção da membrana atlanto-occipital com o osso occipital) houve sucesso quanto à introdução e funcionalidade do cateter para administração de droga e coleta de líquor. A extremidade livre do cateter foi ocluída com um pequeno estilete metálico, para evitar perda de líquor.

O experimento foi feito 24 horas após a colocação do cateter para que não houvesse dúvidas sobre a analgesia residual da anestesia e também para que fosse possível avaliar a ausência de lesão neurológica. Os estudos de literatura também foram realizados somente em animais despertos e movimentando-se livremente, sem contenção ${ }^{4-6}$.

Aimersão da cauda em água quente é um teste que pode ser utilizado em trabalho que utiliza grande número de animais, em que há necessidade de repetição do teste ao longo do experimento para pesquisa de analgesia. Esse teste é sensível e confiável na resposta; é fácil de reproduzir; pode ser executado de maneira rápida; e não provoca lesão térmica. Outros autores também utilizam esse teste ${ }^{11}$.

Neste trabalho foram mergulhados $5 \mathrm{~cm}$ da cauda do animal na água quente, por um período máximo de 15 segundos, para evitar queimaduras, levando-se em conta que o teste foi realizado várias vezes durante o experimento. Nesse experimento, o aquecimento da água do banho-maria foi de $50^{\circ} \mathrm{C}$, porque essa temperatura não é prejudicial ao animal e o efeito do opióide pode ser observado.
Tornou-se evidente a partir de estudos recentes que a imobilização física ou restrição dos movimentos causam estresse em ratos e provocam alterações hormonais e de função do sistema nervoso central. Observa-se ativação do sistema opióide endógeno, resultando em alteração dos testes para analgesia $^{12}$.

Neste estudo foi utilizado um tubo plástico, com pequena abertura na extremidade posterior, para manter exteriorizada a cauda do animal. Não houve necessidade de contenção manual do animal, para a realização do teste, uma vez que os mesmos entraram espontaneamente no cilindro plástico, o que reduz o estresse e impede interferências no resultado do teste de imersão da cauda em água quente.

O presente trabalho utilizou doses pequenas de morfina $(0,1$; 0,$3 ; 0,5 ;$ e $1 \mu \mathrm{g}$ ) para verificar a possibilidade de não ocorrer efeito colateral. Precocemente foram observados agitação em alguns animais e forte estímulo da mandíbula, com rápidos movimentos dos pêlos do focinho e tiritar dos dentes do animal.

Neste estudo foi observada fraqueza das patas em quatro animais logo após a injeção da solução fisiológica; esse efeito não foi provocado pelo opióide, já que não ocorreu após injeção de morfina. Pode estar relacionada à pressão provocada pela solução ${ }^{2}$.

Apesar das buscas na literatura, de trabalhos que justificassem a presença do tremor mandibular observado precedendo o prurido, não foi encontrada nenhuma referência a esse efeito. Entretanto, no presente estudo, o tremor mandibular foi observado, em vários animais de todos os grupos que receberam morfina.

Neste trabalho, o prurido foi o efeito colateral observado com maior freqüência. Esse efeito colateral foi observado clinicamente no focinho e na face de um grande percentual de animais, por aproximadamente duas horas, em concordância com o observado na literatura ${ }^{13}$. Quanto menor a dose utilizada, menor foi à intensidade e a duração do prurido.

Após injeção de morfina marcada por via subaracnóidea lombar de ratos realizando radiografias para identificação da distribuição do opióide, a maior concentração ocorreu após 14 minutos, resultando em prurido no focinho e na face, observado clinicamente até 2 horas após a injeção ${ }^{13}$. Esses autores sugerem que o prurido resultaria de altas concentrações de morfina no nervo trigêmeo. Após administração de morfina sistêmica, o prurido, em parte, envolve o efeito do opióide no sistema nervoso e também pode ser decorrente da liberação de histamina ${ }^{14}$.

Neste estudo, foi observada ausência de diurese em grande número de animais durante algum tempo do experimento e pode estar relacionado à retenção urinária comumente observada em humanos ${ }^{13 .}$

Após a injeção subaracnóidea de morfina, os ratos ficam quietos, com pouca resposta ao manuseio ${ }^{2}$. No presente estudo, foi observado um estado de quietude, em alguns animais, de todos os grupos; considerada sedação leve, pois despertavam facilmente. 
Não foi observada depressão respiratória em nenhum animal investigado, com doses utilizadas neste estudo. As gasometrias arteriais não mostraram alterações, entretanto trata-se de medida isolada, porque foi feita a coleta de sangue de apenas um animal de cada grupo, devido a dificuldades técnicas. Depressão respiratória foi observada em voluntários humanos sadios com 2 a $10 \mathrm{mg}$ de morfina por via peridural ${ }^{5}$. Neste estudo, foi observada alteração respiratória precoce, caracterizada por respiração irregular, mantida até aproximadamente 30 minutos após a injeção de morfina. Não foram observados efeitos colaterais no último tempo de avaliação nos grupos estudados, tal fato pode ser justificado por possível correlação com o tempo transcorrido após a injeção de morfina subaracnóidea. Além disso, foram observados efeitos colaterais mesmo sem analgesia.

Com base nos resultados deste estudo experimental realizado em ratos, com 0,$1 ; 0,3 ; 0,5$ e $1 \mu \mathrm{g}$ de morfina subaracnóidea em $10 \mu \mathrm{l}$ de solução fisiológica a 0,9\%, pode-se concluir que doses menores que $1 \mu \mathrm{g}$ promovem analgesia de curta duração e que não há dose analgésica sem efeito colateral.

\section{Effects of Different Spinal Morphine Doses in Rats}

Neuzimar de Souza Freire Silva, M.D.; Rioko Kimiko Sakata, TSA, M.D.; Adriana Machado Issy, M.D.

\section{INTRODUCTION}

With the understanding of pharmacological properties of analgesics administered by different routes, it became possible to improve pain control, decreasing complications resulting from different painful syndromes.

Experimental investigations have established the basis for the safe and effective use of opioids in men to control acute and chronic pain. After the discovery of opioid receptors in $1974^{1}$, long duration analgesia was obtained with spinal morphine in rats ${ }^{2}$ and men ${ }^{3}$. Since then, these spinal drugs have been frequently used to control acute or chronic pain.

Morphine is a hydrophilic opioid promoting deep and long lasting analgesia without sympathetic or motor block. However, it is cranially spread and may promote side effects such as nausea, vomiting and respiratory failure.

To evaluate the possibility of spinal morphine analgesic effects without side effects, different doses were administered and animals were observed in different moments.

\section{METHODS}

This experimental study was performed after the Research Ethics Committee of the Universidade Federal, São Paulo and Universidade Federal, Amazonas approval and involved 35 Wistar, male, healthy rats aged approximately 90 days and weighing 250 to 300 grams. Excluded from the protocol were animals with paws paralysis or flaccidity. Animals were maintained in individual cages receiving 12 hours of light per day, water and feed ad libitum throughout the experiment.

Animals were distributed in 5 groups of 7 and all have received $10 \mu \mathrm{l}$ spinal solution: $\mathrm{G} 1$ (control) has received $10 \mu \mathrm{l}$ of $0.9 \%$ saline solution; $\mathrm{G} 2$, has received $0.1 \mu \mathrm{g}$ morphine, G3 has received $0.3 \mu \mathrm{g}$ morphine, $\mathrm{G} 4$ has received $0.5 \mu \mathrm{g}$ morphine, and G5 has received $1 \mu \mathrm{g}$ morphine.

Morphine was prepared through the dilution of $1 \mathrm{mg}$ morphine sulfate salt in $1 \mathrm{ml}$ of $0.9 \%$ saline solution $(1 \mathrm{mg} / \mathrm{ml})$. For the $0.1 \mu \mathrm{g} / 10 \mu \mathrm{l}$ solution, $50 \mu \mathrm{l}$ were removed from the stored solution and $4950 \mu \mathrm{l}$ of $0.9 \%$ saline solution were added. For the $0.3 \mu \mathrm{g} / 10 \mu \mathrm{l}$ solution, $100 \mu$ l were removed from the stored solution and $3000 \mu$ l of $0.9 \%$ saline solution were added. For the $0.5 \mu \mathrm{g} / 10 \mu \mathrm{l}$ solution, $100 \mu \mathrm{l}$ were removed from the stored solution and $2000 \mu$ l of $0.9 \%$ saline solution were added. For the $1 \mu \mathrm{g} / 10 \mu \mathrm{l}$ solution, $500 \mu \mathrm{l}$ were removed from the stored solution and $4500 \mu \mathrm{l}$ of $0.9 \%$ saline solution were added.

Animals were submitted to general anesthesia with muscular ketamine $\left(60 \mu \mathrm{g} \cdot \mathrm{g}^{-1}\right)$ and xylazine $\left(16 \mu \mathrm{g} \cdot \mathrm{g}^{-1}\right)$ for spinal catheter insertion by the modified Yaksh, Rudy technique (1976).

Free catheter tip was externalized on the skin between the occipital and high cervical spine and was fixed with polymerized acrylic and nylon thread.

Animals were maintained in individual cages during anesthetic recovery and those with neurological deficits were excluded from the study. Animals were submitted to tail immersion test in hot water at a temperature of $50 \pm 0.2^{\circ} \mathrm{C}$. For the test, animals' tails were marked with red ink $5 \mathrm{~cm}$ above the tip, for immersion. Animals were placed in a plastic semi-transparent cylindrical tube to prevent free movement. Time for tail removal was recorded and tail was not maintained immersed for more than 15 seconds.

After analgesia test in $\mathrm{M}_{0}$ (before morphine), different morphine concentrations were randomly administered 24 hours after spinal catheter insertion. All animals participating in the study were active, moving, receiving free water and feed and totally recovered from anesthesia.

Catheter injection was performed with $10 \mu \mathrm{l}$ Hamilton syringe graduated at $1 \mu \mathrm{l}$, at a rate of $5 \mu \mathrm{l}$ per second.

Analgesia was tested at $\mathrm{M}_{0}=$ before morphine; $\mathrm{M}_{15}=15 \mathrm{~min}$ utes after morphine; $M_{30}=30$ minutes after morphine; $M_{60}=$ 60 minutes after morphine; $\mathrm{M}_{120}=120$ minutes after morphine and $\mathrm{M}_{180}=180$ minutes after morphine.

Possible side effects and complications were recorded at $M_{0}$, $\mathrm{M}_{5}, \mathrm{M}_{15}, \mathrm{M}_{30}, \mathrm{M}_{60}, \mathrm{M}_{120}$ and $\mathrm{M}_{180}$.

Non-parametric tests were used for statistical analysis. Variance Analysis was used for tail immersion test analysis to compare control group in every evaluated time and times within each group. Null hypothesis rejection level for all tests was established as 0.05 or $5 \%(p \leq 0.05)$. 


\section{RESULTS}

\section{Analgesic Effect}

Analgesic effect was not evaluated at $\mathrm{M}_{5}$ in all groups. There have been statistically significant differences in analgesia evaluated by tail removal between animals receiving morphine and control group animals (G1). In G2, time for tail removal was longer as compared to $\mathrm{G} 1$ in $\mathrm{M}_{15}$; in $\mathrm{G} 3$, in $\mathrm{M}_{30}, \mathrm{M}_{60}$ and $\mathrm{M}_{120}$; in $\mathrm{G} 4$ in $\mathrm{M}_{15}, \mathrm{M}_{30}, \mathrm{M}_{60}$ and $\mathrm{M}_{120}$; and in $\mathrm{G} 5$ in $\mathrm{M}_{15}, \mathrm{M}_{30}$, $\mathrm{M}_{60}, \mathrm{M}_{120}$ and $\mathrm{M}_{180}$ (Table I).

Table I - Mean Times for Tail Removal in Groups (G1: control, G2: 0,1 $\mu \mathrm{g}$; G3: 0,3 $\mathrm{gg}$; G4: 0,5 $\mu \mathrm{g}$; G5: $1 \mu \mathrm{g}$ morphine) in Each Moment $\left(M_{0}, M_{15}, M_{30}, M_{60}, M_{120}\right.$ and $\mathrm{M}_{180}$ )

\begin{tabular}{lccccc}
\hline Moments & \multicolumn{5}{c}{ Groups } \\
\hline & $\mathrm{G} 1$ & $\mathrm{G} 2$ & $\mathrm{G} 3$ & $\mathrm{G} 4$ & $\mathrm{G} 5$ \\
\hline $\mathrm{M}_{0}$ & 8.66 & 8.14 & 9.29 & 8.43 & 10.57 \\
$\mathrm{M}_{15}$ & 9.86 & $12.86^{*}$ & 11.71 & $13.14^{*}$ & $14.00^{*}$ \\
$\mathrm{M}_{30}$ & 9.71 & 12.00 & $12.71^{*}$ & $14.86^{*}$ & $15.00^{*}$ \\
$\mathrm{M}_{60}$ & 9.14 & 12.57 & $12.86^{*}$ & $13.29^{*}$ & $13.86^{*}$ \\
$\mathrm{M}_{120}$ & 8.86 & 8.86 & $11.57^{*}$ & $12.43^{*}$ & $12.43^{*}$ \\
$\mathrm{M}_{180}$ & 8.57 & 8.14 & 9.00 & 9.57 & $11.00^{*}$ \\
\hline
\end{tabular}

$\mathrm{M}_{0}: \mathrm{G} 1=\mathrm{G} 2=\mathrm{G} 3=\mathrm{G} 4=\mathrm{G} 5 ; \mathrm{M}_{15}: \mathrm{G} 2, \mathrm{G} 4$ and $\mathrm{G} 5>\mathrm{G} 1=\mathrm{G} 3 ; \mathrm{M}_{30}: \mathrm{G} 3$, $\mathrm{G} 4$ and $\mathrm{G} 5>\mathrm{G} 1=\mathrm{G} 2 ; \mathrm{M}_{60}: \mathrm{G} 3, \mathrm{G} 4$ and $\mathrm{G} 5>\mathrm{G} 1=\mathrm{G} 2 ; \mathrm{M}_{120}: \mathrm{G} 3, \mathrm{G} 4$ and $\mathrm{G} 5>\mathrm{G} 1=\mathrm{G} 2 ; \mathrm{M}_{180}: \mathrm{G} 5>\mathrm{G} 1=\mathrm{G} 2=\mathrm{G} 3=\mathrm{G} 4$.

${ }^{*}=$ statistical significance; Variance Analysis $(p \leq 0.05)$

G2 presented analgesic effects in $85.72 \%$ of rats in $\mathrm{M}_{15}$; and $71.43 \%$ in $\mathrm{M}_{30}$ and $\mathrm{M}_{60}$. In $\mathrm{G} 2,71.43 \%$ in $\mathrm{M}_{15} ; 100 \%$ in $\mathrm{M}_{30}$ and $M_{60}$ and $14.28 \%$ in $M_{120}$. In $G 4,100 \%$ of animals in $M_{15}, M_{30}$, $\mathrm{M}_{60} ; 71.43 \%$ in $\mathrm{M}_{120}$. In $\mathrm{G} 5,100 \%$ in $\mathrm{M}_{15}, 85.72 \%$ in $\mathrm{M}_{30}$ and $100 \%$ in $\mathrm{M}_{60}$.

There were no statistically significant differences in mean times for tail removal in $\mathrm{M}_{0}, \mathrm{M}_{15}, \mathrm{M}_{30}, \mathrm{M}_{60}, \mathrm{M}_{120}$ and $\mathrm{M}_{180}$ in the control group. In groups 2, 3 and 5, in $M_{15}, M_{30}$ and $M_{60}$ they were longer than in $M_{120}$ and $M_{180}$, which were similar to $M_{0}$. In $G 4$, in $M_{15}, M_{30}, M_{60}$ and $M_{120}$ they were longer than in $M_{180}$, which was similar to $M_{0}$. Variance Analysis, $p \geq 0.05$ (Table II).

Table II - Mean Times for Tail Removal During Different Periods in Each Group (G1: control, G2: $0.1 \mu \mathrm{g}$; G3: 0.3 $\mu \mathrm{g} ; \mathrm{G} 4: 0.5 \mu \mathrm{g}$; G5: $1 \mu \mathrm{g}$ morphine)

\begin{tabular}{lcccccc}
\hline Groups & \multicolumn{5}{c}{ Moments after Solutions Administration } \\
\hline & $M_{0}$ & $M_{15}$ & $M_{30}$ & $M_{60}$ & $M_{120}$ & $M_{180}$ \\
\hline G1 $(n=7)$ & 8.66 & 9.86 & 9.71 & 9.14 & 8.86 & 8.57 \\
G2 $(n=7)$ & 8.14 & $12.86^{*}$ & $12.00^{*}$ & $12.57^{*}$ & 8.86 & 8.14 \\
G3 $(n=7)$ & 9.29 & $11.71^{*}$ & $12.71^{*}$ & $12.86^{*}$ & 11.57 & 9.00 \\
G4 (n=7) & 8.43 & $13.14^{*}$ & $14.86^{*}$ & $13.29^{*}$ & $12.43^{*}$ & 9.57 \\
G5 $(n=7)$ & 10.57 & $14.00^{*}$ & $15.00^{*}$ & $13.86^{*}$ & 12.43 & 11.00 \\
\hline
\end{tabular}

$G 2: M_{15}, M_{30}$ and $M_{60}>M_{0}=M_{120}=M_{180} ; G 3: M_{15}, M_{30}$ e $M_{60}>M_{0}=M_{120}=$ $M_{180} ; G 4: M_{15}, M_{30}, M_{60}$ e $M_{120}>M_{0}=M_{180} ; G 5: M_{15}, M_{30}$ e $M_{60}>M_{0}=M_{120}$ $=\mathrm{M}_{180}$;

*: statistical significance; Variance Analysis $(p \leq 0.05)$

\section{Side Effects Observed after Injections}

There have been no effects in $\mathrm{G} 1$, except for $\mathrm{M}_{5}$ where there has been paws weakness in four animals (57.15\%).

Agitation was observed in $\mathrm{G} 2$ in $14.28 \%$ of animals in $\mathrm{M}_{15}$; in G3 in $14.28 \%$ in $M_{15}$ and $M_{30}$. There has been mandible tremor in $\mathrm{G} 2$ in $85.72 \%$ of animals in $\mathrm{M}_{5} ; 71.43 \%$ in $\mathrm{M}_{15}$ and $14.28 \%$ in $M_{30}$ and $M_{60}$. This side effect was observed in $100 \%$ of $\mathrm{G} 3$ animals in $\mathrm{M}_{5}$ and in $14.28 \%$ in $\mathrm{M}_{15}$. In $\mathrm{G} 4$, it has been observed in $85.72 \%$ of animals in $\mathrm{M}_{5}$. In $\mathrm{G} 5$ it has been observed in $85.72 \%$ of animals in $\mathrm{M}_{5}$.

There has been pruritus in $\mathrm{G} 2$ in $57.15 \%$ of animals in $\mathrm{M}_{5}$; $71.43 \%$ in $M_{15} ; 57.15 \%$ in $M_{30}$; and $42.86 \%$ in $M_{60}$. In $G 3$, this effect was observed in $42.86 \%$ in $\mathrm{M}_{5}, 85.72 \%$ in $\mathrm{M}_{15} ; 100 \%$ in $M_{30}$ and $M_{60}$ and $57.15 \%$ in $M_{120}$. In $G 4$, it has been observed in $85.72 \%$ in $M_{5}, 100 \%$ of animals in $M_{15}, M_{30}$ and $M_{60}$ and $71.43 \%$ in $M_{120}$. No $G 5$, it has been observed $71,43 \%$ pruritus in $M_{5}, 100 \%$ in $M_{15}, M_{30}, M_{60}$ and $28,58 \%$ in $M_{120}$.

There has been absence of diuresis in $\mathrm{G} 2$ in $85.72 \%$ of animals in $M_{60}$. In G3, this effect has been observed in $14.28 \%$ in $\mathrm{M}_{15}$ and $\mathrm{M}_{30} ; 71.43 \%$ in $\mathrm{M}_{60}$ and $28.58 \%$ in $\mathrm{M}_{120}$. In $\mathrm{G} 4$, there has been absence of diuresis in $85.72 \%$ in $M_{60}$ and $28.58 \%$ in $\mathrm{M}_{120}$. In $\mathrm{G} 5$, absence of diuresis has been observed in $14.28 \%$ of animals in $\mathrm{M}_{30}$ and $100 \%$ in $\mathrm{M}_{60}$.

Sedation was observed in $G 2$ in $100 \%$ of animals in $M_{15}$, $85.72 \%$ in $M_{30}$ and $57.15 \%$ in $M_{60}$. In G3, the same effect has been observed in $57.15 \%$ in $M_{15}, 85.72 \%$ in $M_{30}$ and $57.15 \%$ in $M_{60}$. In G4, sedation was observed in $100 \%$ of animals in $M_{15}$ and $\mathrm{M}_{30}$ and $71.43 \%$ in $\mathrm{M}_{60}$. In $\mathrm{G} 5$, sedation has been observed in $100 \%$ of animals in $M_{15}$, and $M_{30}$, in $57.15 \%$ in $M_{60}$ and $14.28 \%$ in $\mathrm{M}_{120}$.

There has been respiratory changes in $\mathrm{G} 2$ in $71.43 \%$ of animals in $\mathrm{M}_{15}$ and $\mathrm{M}_{30}$, and $42.86 \%$ in $\mathrm{M}_{60}$. In $\mathrm{G} 3$, there has been respiratory changes in $42.86 \%$ of animals in $M_{15}, 14.28 \%$ in $M_{30}$ and $M_{60}$. In G4, this effect has been observed in $100 \%$ of animals in $\mathrm{M}_{15}$ and $\mathrm{M}_{30}$. In $\mathrm{G} 5$, respiratory changes have been observed in $42.86 \%$ in $M_{15}$ and $57.15 \%$ in $M_{30}$.

\section{DISCUSSION}

Wistar rats were used in this study due to their fast reproduction and small size, making easy their handling and the maintenance of hygiene and food in the cages. They are also very resistant to infections and have low cost. Several authors have also chosen rats for spinal morphine injections, due to the above-described reasons ${ }^{4,5}$.

Morphine was the opioid of choice for being a potent spinal analgesic drug, especially to treat acute pain and chronic painful syndromes. For being hydrophilic, it has prolonged action time, but this characteristic is also responsible for longer CSF permanence, as compared to lypophilic opioids. This leads to more cranial spread and opioid binding to brain receptors promoting side effects such as pruritus, nausea, vomiting and respiratory depression. These side effects limit the use of spinal opioids; so, if there could be the possibility of administering a dose producing analgesic effect without side effects its use could be expanded. 
First investigations have questioned whether any volume of opioids would reach brain structures immediately after injection. Initially, it was thought that the drug would move in the cranial direction passively following CSF movement within the spinal space. After the administration of different bromphenol blue volumes in the spinal space of rats followed by spinal cord analysis ${ }^{4}$, authors have concluded that $10 \mu \mathrm{l}$ is the most adequate volume. Other authors have used 5 to 20 $\mu \mathrm{l}^{6}$, up to $100 \mu \mathrm{l}^{7}$ epidural and spinal volumes. An author, however, has compared different spinal volumes and has concluded that there has been a better spread with $10 \mu \mathrm{l}$, even stating that large volumes would promote losses by reflux and by the lateral sides of the catheter ${ }^{8}$. Studies have shown no need for high spinal morphine volumes to obtain analgesic effects. As from the above-mentioned studies, we decided for $10 \mu \mathrm{l}$ for this study, for being a volume with better drug spread uniformity within the spinal space, and for safety reasons.

Doses of 0.1 to $1 \mu \mathrm{g}$ were chosen because a study has shown that $1 \mu \mathrm{g}$ spinal morphine produces analgesic effects ${ }^{8}$, but also promotes side effects; so, we had to use lower doses to check whether there are analgesic effects without side effects.

Some authors have evaluated extracellular morphine concentrations in spinal cord, peri-aqueduct gray matter, raphe nucleus and lateral hypothalamus after 5 and $10 \mathrm{mg}$ intraperitoneal morphine. In our study, morphine doses have promoted analgesia in several studied moments, however in most moments, morphine CSF concentration was lower than the minimum $200-400 \mathrm{pg} / 100 \mu \mathrm{l}$ concentration reported in the literature ${ }^{9}$.

We decided for silicone rubber catheters for being flexible, inert and nontoxic, not promoting tissue inflammatory reactions. Such features were confirmed by spinal cord histopathological studies in rats which remained with silicone rubber catheters for four weeks ${ }^{10}$. Small catheters were used in this study to prevent spinal cord compression.

Modified Yaksh, Rudy technique ${ }^{4}$ was used for catheter insertion, for allowing relatively easy spinal space access via cisterna magna. It also allows for catheter maintenance for prolonged periods because they can be effectively fixed, in addition to being isolated from animal's mouth and paws, thus preventing removal. This method has been successfully used to study the efficacy of spinal agents. However, if strictly reproduced, it demands a very long time for catheter insertion with high CSF collection failure rate.

Initial transmembrane puncture was performed according to authors' recommendations, with puncture in the middle of the atlanto-occipital membrane, but there has been failure in serial CSF collection. With the modification and the catheter more laterally inserted (atlanto-occipital membrane and occipital bone junction) there has been successful catheter insertion and functionality for drug administration and CSF collection. Free catheter tip was occluded with a small metal probe to prevent CSF leakage.

The experiment was performed 24 hours after catheter insertion to eliminate issues on residual anesthetic analgesia, and also to provide the evaluation of no neurological injuries. Studies in the literature were also just performed in awaken animals moving freely without contention ${ }^{4-6}$.

Tail immersion in hot water is a test which may be performed with a large number of animals when there is the need for repeating the test along the experiment to evaluate analgesia. It is sensitive, reliable and easy to reproduce. It is fast and does not induce heat injury. Other authors have also used this test ${ }^{11}$.

In this study, 5 centimeters of tail were immersed in hot water for a maximum period of 15 seconds to prevent burns, taking into account that the test has been performed several times during the experiment. Water was heated in double boiler at $50^{\circ} \mathrm{C}$ because this temperature is not deleterious to animals and allows for the observation of opioid's effects.

It has been apparent from recent studies that physical immobilization or movement restriction stresses rats and promotes hormonal and central nervous system changes. Endogenous opioid system is activated and changes analgesia tests results ${ }^{12}$.

Our study has used plastic tubes with small posterior edge opening to maintain animal's tail externalized. There has been no need for manual contention of animals for the test because they have spontaneously entered the plastic cylinder, thus decreasing stress and preventing interferences in tail immersion test in hot water results.

We have used low morphine doses $(0.1,0.3,0.5$ and $1 \mu \mathrm{g})$ to check the possibility of no side effects. Agitation and strong mandible stimulation with fast muzzle hair movements and teeth shivering were earlier observed in some animals.

Paws weakness was observed in 4 animals soon after saline injection; this was not an opioid-induced effect since it was not seen after morphine injection, but could be related to solution-induced pressure ${ }^{2}$.

No reference was found in the literature to justify the presence of mandible tremor preceding pruritus. However, mandible tremor was observed in our study in several animals of all groups receiving morphine.

Pruritus was the most common side effect. It has been clinically observed on muzzle and face of a large number of animals for approximately 2 hours and in line with the literature ${ }^{13}$. The lower the dose used, the milder the intensity and duration of pruritus.

After labeled lumbar spine morphine in rats with X-rays to identify opioid distribution, highest concentration was observed 14 minutes after, resulting in muzzle and face pruritus clinically observed up to 2 hours after injection ${ }^{13}$. These authors have suggested that pruritus would result from high morphine concentration on the trigeminal nerve. After systemic morphine administration, pruritus partially involves the opioid effect on nervous system and may also be a consequence of histamine release ${ }^{14}$.

There has been absence of diuresis in our study in a large number of animals for some time and this may be related to urinary retention commonly observed in men ${ }^{13}$.

After spinal morphine administration, rats remain quiet, with low response to handling ${ }^{2}$. In our study, quietness has been Vol. 54, N 1, Janeiro - Fevereiro, 2004 
observed in some animals of all groups and was considered mild sedation since they were easily awakened.

There has been no respiratory depression in all studied animals with the doses used in our study. Arterial blood gases analysis has not shown changes, however this was an isolated measurement because blood was collected from one animal of each group only, due to technical difficulties. Respiratory depression has been observed in human healthy volunteers with 2 to $10 \mathrm{mg}$ epidural morphine ${ }^{5}$. Our study has observed early respiratory changes characterized by irregular breathing maintained for approximately 30 minutes after morphine injection.

There have been no side effects in the last evaluation moment in all groups and this might be justified by a possible correlation with time elapsed after spinal morphine injection. In addition, side effects were observed even without analgesia. Based on this experimental study with rats and $0.1,0.3,0.5$ and $1 \mu \mathrm{g}$ spinal morphine in $10 \mu \mathrm{l}$ of $0.9 \%$ saline solution, one may conclude that doses below $1 \mu \mathrm{g}$ promote short duration analgesia and that there is no analgesic dose without side effects.

\section{REFERÊNCIAS - REFERENCES}

01. Snyder SH, Pert CB, Pasternak GW - The opiate receptor. Ann Intern Med, 1974;81:534-540.

02. Yaksh TL, Rudy TA - Studies on direct spinal action of narcotic in the production of analgesia in the rat. J Pharmacol Exp Ther, 1977;202:411-428.

03. Wang JK, Nauss LA, Thomas JE - Pain relief by intrathecally applied morphine in man. Anesthesiology, 1979;50:149-151.

04. Yaksh L, Rudy TA - Chronic catheterization of the spinal subarachnoid space. Physiol Behav, 1976;17:1031-1036.

05. Van den Hoogen RGW, Colpaert FC - Long term catheterization of the lumbar epidural space in rats. Pharmacol Biochem Behav, 1981;15:515-516.

06. Dib B - Intrathecal chronic catheterization in the rat. Pharmacol Biochem Behav, 1984;20:45-48.

07. Immelman L, Roth S, Sabourin MA et al - Analgesia and serum concentrations of extradural, subdural and intraperitoneal fentanyl in a rat model. Can J Anaesth, 1990;37:63-68.

08. Nishiyama T - A rat model of chronic lumbar epidural catheterization. Can J Anaesth, 1998;45:907-912.

09. Matos FF, Rollema H, Taiwo Y et al - Relationship between analgesia and extracelular morphine in brain and spinal cord in awake rats. Brain Research, 1995;693:187-195.

10. Bahar M, Rosen M, Vickers MD - Chronic cannulation of the intradural or extradural space in the rat. Br J Anaesth, 1984;56: 405-410.
11. Janssen PAJ, Niemeregeers CJE, Dony JG - The inhibitory effect of fentanyl and other dinorphine-like analgesics on the warm water induced tail withdrawal reflex in rats. Arzneimittel-Forsch, 1963;13:502-507.

12. Porro CA, Carli G - Immobilization and restraint effects on pain reactions in animals. Pain, 1988;32:289-307.

13. Gustafsson LL, Post C, Edvardsen B et al - Distribution of morphine and meperidine after intrathecal administration in rat and mouse. Anesthesiology, 1985;63:484-489.

14. Jaffe JH, Martin WR - Opioid Analgesics and Antagonists, em: Goodman \& Gilman's - The Pharmacological Basis of Therapeutics. $8^{\text {th }}$ Ed, New York: Pergamon Press, 1990;485-521.

\section{RESUMEN}

Silva NSF, Sakata RK, Issy AM - Efectos Observados con Diferentes Dosis de Morfina Subaracnoidea en Ratones

JUSTIFICATIVA Y OBJETIVOS: La morfina por vía espinal promueve buen efecto analgésico, solamente no es exenta de efecto colateral. El objetivo de este estudio fue investigar los efectos observados con diferentes dosis de morfina por via subaracnoidea.

MÉTODO: Fueron estudiados cinco grupos de siete ratones, 24 horas después de la colocación de catéter subaracnoideo vía cisterna magna bajo anestesia con cetamina y xilazina por la vía muscular. El G1 recibió $10 \mu$ l de solución fisiológica; los grupos G2, G3, G4, y G5 recibieron respectivamente 0,1; 0,3; 0,5 y $1 \mu \mathrm{g}$ de morfina en $10 \mu \mathrm{l}$ de solución fisiológica. Los animales fueron sometidos al teste de inmersión de la cola en agua caliente en el $M_{0}$ (antes de la inyección), y $M_{15}, M_{30}, M_{60}$, $M_{120}$ y $M_{180}$ minutos después de la inyección.

RESULTADOS: Se observó analgesia en los grupos que recibieron morfina, en diversos momentos, cuando comparados con el grupo control y con el tiempo antes de la inyección de morfina. En el G1 hubo flaqueza de las patas en 4 animales. Ocurrió agitación en $M_{15}$ en el G2 y en $M_{15}$ y $M_{30}$ en el G3. Tremor de mandíbula fue observado en $M_{5}, M_{15}, M_{30}$, y $M_{60}$ en el G2; en el G3 fue observado en $M_{5}$ y $M_{15}$; en el G4, en $M_{5} y$ en el G5, en $M_{5}$. Prurito fue observado en $M_{5}, M_{15}, M_{30}$ y $M_{60}$ en el G2; en $M_{5}, M_{15}, M_{30}, M_{60}$ y $M_{120}$ en el G3; en $M_{5}, M_{15}, M_{30}, M_{60}$, y $M_{120}$ en el G4; en $M_{5}, M_{15}, M_{30}, M_{60}$ y $M_{120}$ en el G5. Ausencia de diúresis ocurrió en $M_{60}$ en el G2; en el $M_{15}, M_{30}, M_{60}$ y $M_{120}$ en el G3; en el $M_{60}$ y $M_{120}$ en el G4; y en el $M_{30}$ y $M_{60}$ en el G5. Sedación ocurrió en $M_{15}, M_{30}$ y $M_{60}$ en los grupos 2, 3 y 4; y en $M_{15}, M_{30}, M_{60}$, y $M_{120}$ en el G5. Alteración respiratoria fue observada en $M_{15}, M_{30}, M_{60}$ en el G2; en $M_{15}, M_{30}$ y $M_{60}$ en el G3; en $M_{15}$ y $M_{30}$ en los grupos 4 y 5 .

CONCLUSIONES: En este estudio, todas las dosis de morfina subaracnoideas administradas provocaran efectos colaterales; $y$ dosis menores que $1 \mu g$ promovieron analgesia de corta duración. 